\title{
EVALUACIÓN CLÍNICA DE LA RECONSTRUCCIÓN ORBITARIA POST TRAUMÁTICA MEDIANTE MALLAS DE TITANIO*
}

\author{
Drs. Edgardo González M. ${ }^{1}$, Christian Pedemonte T. ${ }^{1}$, \\ Ilich Vargas F. ${ }^{1}$, Francisco Verdugo-Avello ${ }^{1}$ \\ 1 Unidad de Cirugía Maxilofacial, Policlínico de Especialidades, Hospital Clínico Mutual de Seguridad. \\ Santiago, Chile.
}

\begin{abstract}
Clinical assessment of post traumatic orbital reconstruction by titanium mesh

Objectives: Collect maxillofacial trauma data, specifically in orbital reconstruction, in adult patients to make an evaluation of the clinical results. Material and Methods: Reviewing medical records in a three years period (January 2009-December 2011), of patients with orbital fracture reconstruction who attended the Maxillofacial Surgery Service of Hospital Mutual de Seguridad, Chile, in a retrospective study. Results: There where a total of 87 patients with 88 orbital fractures diagnosis, 30 patients go to orbital reconstruction with orbital titanium mesh. The most common cause where accidents and violence traffic. It was observed a $6.7 \%$ of complications. Discussion: The orbital fracture pattern and the results cause diplopia as the main complication. Furthermore, we can conclude that the orbital titanium mesh is an effective material and allows a well adaptation and lower complications.
\end{abstract}

Key words: Facial trauma, orbital fractures, titanium orbital mesh.

\section{Resumen}

Objetivo: Obtener información del trauma maxilofacial, específicamente de la reconstrucción orbitaria, en pacientes adultos para la evaluación de sus resultados clínicos. Materiales y Métodos: Se realizó un estudio descriptivo retrospectivo en todos los casos de fracturas orbitarias que asistieron al servicio de cirugía maxilofacial del hospital clínico Mutual de Seguridad de Santiago de Chile. En el período de 3 años (enero de 2009-diciembre de 2011). La necesidad de reconstrucción fue el criterio de inclusión. Resultados: Se registraron 87 pacientes con 88 diagnósticos de fracturas orbitarias, de estos, 30 pacientes debieron someterse a reconstrucción orbitaria mediante malla de titanio. Las causas más comunes fueron el accidente de tránsito $34 \%$ y las agresiones 30\%. Se observó una tasa de complicaciones de 6,7\%. Discusión: El patrón de fractura orbitaria observado y nuestros resultados muestran a la diplopía como principal complicación, además podemos señalar que las mallas de titanio son un material eficaz que permite una apropiada adaptación y bajas complicaciones.

Palabras clave: Trauma facial, fracturas orbitarias, mallas orbitarias de titanio.

*Recibido el 23 de junio de 2013 y aceptado para publicación el 4 de agosto de 2014.

No obtuvimos financiamiento o ayuda financiera para realizar este trabajo.

Correspondencia: Dr. Francisco Verdugo-Avello

Verdugo_fco@hotmail.com

Dr. Edgardo González M.

edggonzalez@mutual.cl 


\section{Introducción}

Las fracturas orbitarias son un componente específico del trauma en el tercio medio facial, presentándose de manera aislada o asociadas a otra estructura ósea. Representan aproximadamente un $40 \%$ del trauma craneofacial ${ }^{1}$. El piso y la pared medial de la órbita son las más propensas a fracturarse gracias a sus características de grosor y falta de sostén ${ }^{2}$.

Las indicaciones quirúrgicas pueden ser estéticas y/o funcionales, siendo la restauración de la configuración de las paredes orbitarias la meta de la reconstrucción primaria, devolviendo el contenido orbitario colapsado y eliminando cualquier impedimento o atrapamiento del tejido blando ${ }^{3,4}$.

Esta patología traumática sigue siendo una de las reconstrucciones más complejas y desafiantes en la cirugía maxilofacial ${ }^{5}$. Hoy en día algunas técnicas novedosas como el prototipo rápido y modelos estereolitográficos son más accesibles ${ }^{5-8}$.

En la literatura se describen diferentes métodos quirúrgicos, así como también distintos materiales para la reconstrucción orbitaria. Sin embargo, los resultados terapéuticos no son siempre satisfactorios, ya que pueden ocurrir muchas complicaciones postoperatorias 9 .

Existe una abundante disponibilidad de materiales para solucionar la difícil tarea de la reconstrucción $^{10}$. Entre los biomateriales se incluyen los naturales y sintéticos. Los que pueden ser clasificados en aloplásticos, aloinjertos, autoinjertos y xenoinjertos. Presentando un rango de complicaciones que varían entre $0,5 \%$ y $85,5 \% \%^{9,11,12}$.

La malla de titanio es usada de manera rutinaria en el tratamiento de fracturas orbitarias y ha mostrado un buen éxito cuando es usada de manera apropiada ${ }^{13,14}$. Muchos investigadores concuerdan que esta malla de titanio es una opción eficaz para la reparación de rutina de las fracturas de piso orbitario $^{15-18}$. No obstante, estas mallas también presentan cierto riesgo, ya que en los casos que se requiera una segunda intervención, su remoción podría ser muy difícil por las fuertes adhesiones del tejido periorbitario a la malla ${ }^{19}$.

El objetivo del presente estudio descriptivo es recopilar información del trauma maxilofacial, específicamente de la reconstrucción orbitaria, en pacientes adultos para la evaluación de sus resultados clínicos.

\section{Materiales y Métodos}

Se realizó un estudio descriptivo retrospectivo en todos los casos de fracturas orbitarias que asistieron al servicio de cirugía maxilofacial del hospital clínico Mutual de Seguridad de Santiago de Chile.
En un período de 3 años (enero de 2009-diciembre de 2011). Todas las fracturas orbitarias fueron consignadas. La información fue recopilada a través de revisión de fichas clínicas electrónicas de manera sistemática por un solo investigador.

Las causas de las fracturas orbitarias fueron clasificadas en cinco categorías: caídas, golpe con objeto (herramienta o material industrial), accidente de tráfico (motocicleta, automóvil, bicicleta y atropello), violencia y otros. Fueron incluidos todos los pacientes con alguna fractura de pared orbitaria con tratamiento abierto y requerimiento de reconstrucción. La locación orbitaria fue dividida en pared medial, piso orbitario y techo orbitario (fracturas orbitarias puras). Las fracturas de la pared lateral de la órbita fueron excluidas, ya que son consideradas como fractura del complejo cigomáticomaxilar.

La frecuencia, tipo de fractura y causa del daño, edad de distribución y género, así como también las complicaciones fueron analizadas. Las comparaciones fueron realizadas mediante test $\chi^{2}$. Esto fue seguido por un análisis de regresión logística en caso de haber diferencias significativas entre las variables.

La secuencia de evaluación fue la siguiente: Una vez presentado el caso se realiza un examen clínico y evaluación de daño ocular, si es posible se ejecutan test orbitarios, evaluación de agudeza visual, reflejos fotomotor, consensual, motilidad ocular y evaluación de presencia de "defecto pupilar aferente relativo". Interconsulta a oftalmología para descartar daño ocular. Toma de tomografía computada con cortes finos 0,6-1 mm de la zona orbitaria.

Las indicaciones quirúrgicas establecidas en el servicio son diplopía inmediata previa a edema, restricción franca de la motilidad por atrapamiento muscular, "white eye blow out syndrome" (escasos signos clínicos y radiográficos donde ocurre un atrapamiento muscular, principalmente en pacientes pediátricos), reflejo óculo-cardíaco persistente, compresión a nervio óptico (hematoma, fragmento óseo), diplopía post-edema invalidante y enoftalmo con compromiso estético.

Una vez confirmado el diagnóstico y descartado el trauma ocular, se realiza la cirugía bajo anestesia general. Comúnmente se realiza un abordaje transconjuntival retroseptal con cantotomía lateral para la mejor visualización del piso orbitario posterior a un decolamiento subperióstico. Si se acompaña de requerimiento de reconstrucción de la pared medial se realiza un abordaje retrocaruncular. En algunas ocasiones se usa también el acceso palpebral inferior. Una vez visualizado el defecto óseo se liberan los atrapamientos musculares y se devuelve el contenido herniado de los senos paranasales hacia la órbita. Se usa el canal infraorbitario y la porción orbitaria del 
hueso palatino como referencias. Después de esto se procede a la colocación de la malla orbitaria de titanio, teniendo apoyo óseo (en hueso no fracturado) de los límites de ésta en lo posible, asegurando además, que no provoque un atrapamiento de tejido blando que después restrinja los movimientos oculares. Finalizamos con un test de ducción forzada.

Como objetivo de la reconstrucción se plantea devolver la anatomía perdida, con especial atención en la eminencia retrobulbar y lograr una adecuada proyección ocular.

Se realiza una tomografía computada postoperatoria para evaluar la posición de la malla, y controles periódicos a los 7 días, 15 días, 30 días, 3 meses con evaluación clínica de los resultados mediante examen de motilidad ocular y estético (para descartar un posible enoftalmo postoperatorio).

Dadas las características retrospectivas del estudio, la aprobación del comité de ética hospitalario fue requerida y aceptada.

\section{Resultados}

Dentro del período de estudio se registraron 283 pacientes con fracturas faciales, dentro de los cuales se consignaron 87 pacientes con 88 diagnósticos de fracturas orbitarias (un paciente presentó fractura orbitaria bilateral). De estos, 64 pacientes tuvieron indicación quirúrgica con elementos de osteosíntesis por alguna fractura asociada al de la órbita (principalmente fracturas del tercio medio facial) sin requerimiento de reconstrucción mediante malla de titanio. Dentro de ellos, 30 pacientes debieron someterse a reconstrucción orbitaria mediante malla de titanio, las 34 fracturas restantes no requirieron colocación de algún elemento de osteosíntesis dentro de la órbita.

En cuanto a los datos epidemiológicos, 78 pacientes fueron hombre y 9 mujeres. Edad promedio del grupo fue 39 años siendo el rango más prevalente de la década de los 40 a los 49 años. El seguimiento promedio de los pacientes fue de 4,9 meses.

La localización de las fracturas se encontró principalmente en el piso orbitario, en 51 ocasiones, pared medial 14 , techo 5 . La combinación más frecuente fue la de la pared medial con el piso orbitario en 13 ocasiones, sólo 2 pacientes tuvieron afectadas las 3 paredes de la órbita.

Las causas más comunes fueron el accidente de tránsito $34 \%$ y las agresiones $30 \%$, seguido por las caídas $17 \%$ y golpe con objeto $14 \%$. Dentro de los accidentes de tránsito el principal agente causal fue el accidente automovilístico con un 62\% (Figura 1).

La evaluación preoperatoria mostró que la diplopía es el signo más prevalente en 48 ocasiones, seguido del enoftalmo en 9, hipoftalmo en 6 y 2 lesiones musculares al recto inferior.

La reconstrucción quirúrgica fue realizada principalmente en base al acceso transconjuntival (20/30 veces), transconjuntival con retrocaruncular 6 ocasiones, subpalpebral 2 veces y sólo en una ocasión se pudo usar la exposición del área a reconstruir a través de la herida del trauma. Todos los accesos transconjuntivales fueron retroseptales con cantotomía lateral (Figuras 2 y 3 ). En cuanto a las mallas de titanio se utilizó en su mayoría malla de titanio preformada (Preformed Matrix Orbital Mesh ${ }^{\circledR}$, Synthes), en un 58\%; también se usaron las mallas de titanio no preformadas (Orbital Floor Mesh ${ }^{\circledR}$, KLS Martin), en un $42 \%$. La elección de cada una fue dependiente de las preferencias del cirujano, sin objetivo de comparación.

Se consignaron 10 complicaciones postoperatorias inmediatas, 3 enoftalmos (Figura 4) y 7 diplopías. Dentro de las diplopías, 4 fueron de resolución

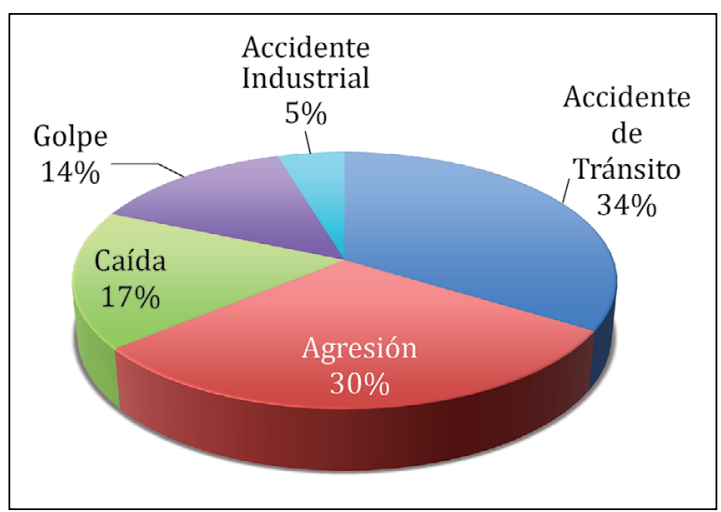

Figura 1. Gráfico que muestra las causas de las fracturas orbitarias. Notar la alta presencia de etiología por accidente de tránsito y agresiones.

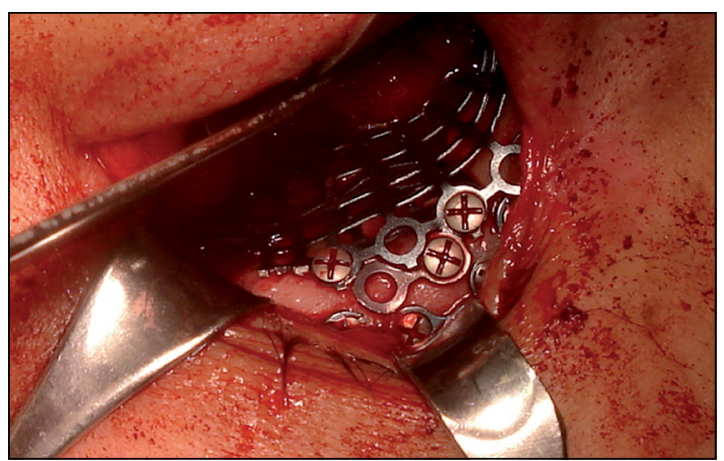

Figura 2. Acceso transconjuntival con cantotomía lateral donde se aprecia la colocación de una malla de titanio preformada y fijada con elementos de osteosíntesis. 


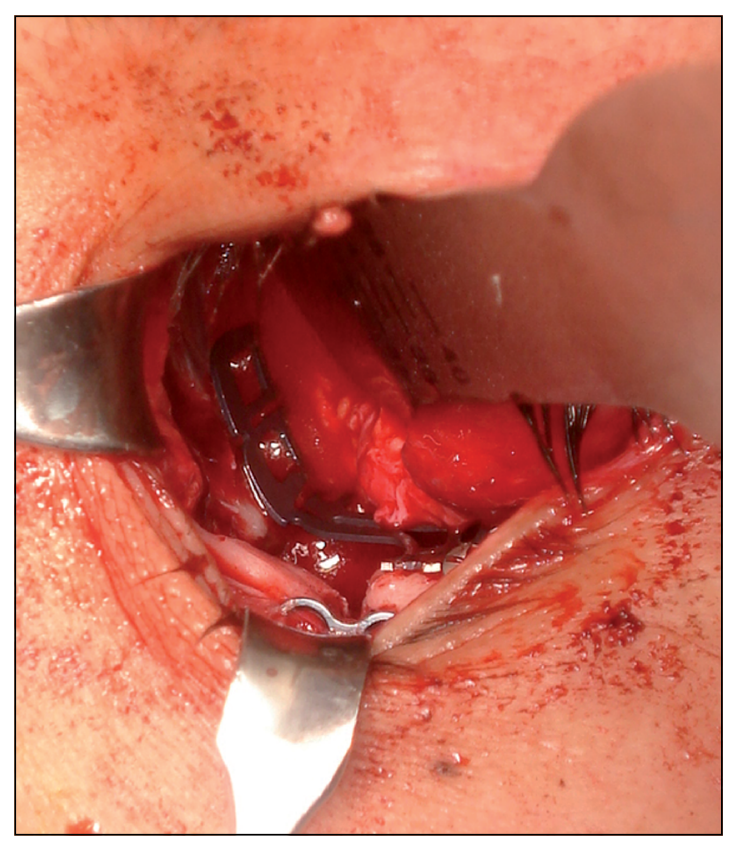

Figura 3. Acceso transconjuntival asociado con un acceso transcaruncular, donde se aprecia la exposición de la pared medial con una malla preformada en posición.

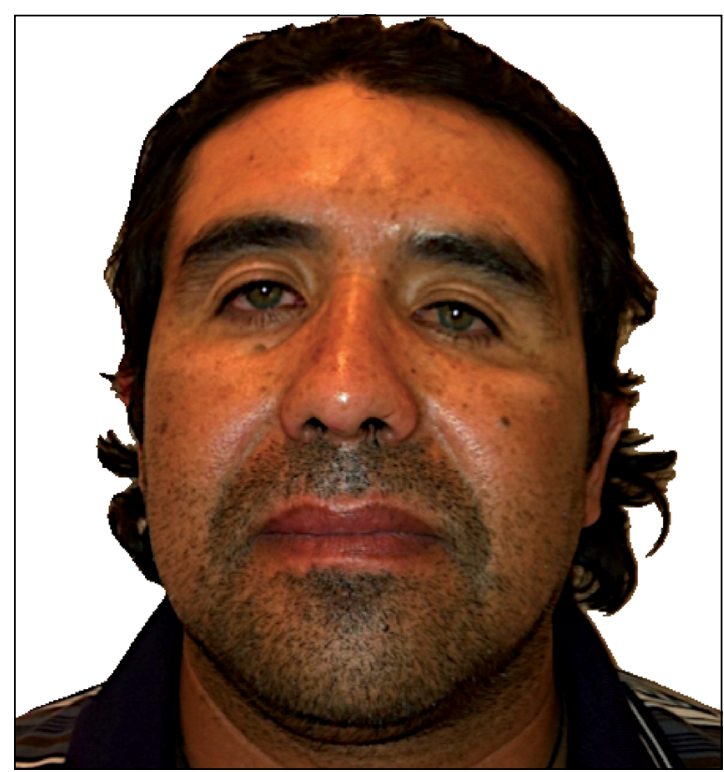

Figura 4. Paciente con enoftalmo e hipoftalmo izquierdo.

kinésica quedando sin alteraciones al alta. Dos daños musculares por el trauma que no fueron pesquisados en primera instancia, se resolvieron con una segunda intervención por parte del oftalmólogo, con resolución del problema. Sólo 2 pacientes quedaron con diplopía leve al alta, compatibles con su reinserción laboral. Dentro de los 3 enoftalmos, uno fue por causa de falta de proyección cigomática, la que fue resuelta en una segunda intervención quirúrgica. Los otros 2 pacientes no quisieron someterse a una segunda intervención, por lo que se consignan éstos como 2 secuelas finales, es decir, una tasa de complicación tardía del $6,7 \%$. No se encontraron complicaciones inherentes a las vías de acceso ni al tipo de malla utilizada.

\section{Discusión}

Este análisis retrospectivo fue realizado con 87 pacientes que sufrieron fractura orbitaria dentro de los cuales 30 requirieron reconstrucción de la órbita mediante mallas de titanio. El objetivo primario de esta reconstrucción quirúrgica es la liberación del tejido blando atrapado y restaurar la anatomía al igual que su volumen original ${ }^{20-22}$. Hasta hoy, la elección del mejor material para realizar la reconstrucción sigue en discusión.

Las ventajas y desventajas de todo material reportado está bien documentada en la literatura, pero el material óptimo y que logre consenso para la reconstrucción orbitaria, aún es tema controversial y de debate. Los materiales más usados son los autógenos, alogénicos y aloplásticos ${ }^{15}$.

Durante los últimos 20 años, el uso de las mallas de titanio en las fracturas orbitarias ha incrementado su uso, reportando buenos resultados. Al principio se usaban para aumentar la estabilidad del injerto óseo, pero después demostraron que podían ser mucho más precisas en la reconstrucción tridimensional de la órbita, especialmente en la región posteromedial. El diseño de la malla de titanio también ha evolucionado a formas más específicas y adaptables, lo que facilita de gran manera su manipulación e inserción dentro de la compleja anatomía tridimensional de la órbita ${ }^{5,11,15,16,23-29}$. Gracias a esta evolución en la aplicación clínica, elegimos la malla de titanio, específicamente la malla preformada. Hasta el momento no hemos reportado complicaciones inherentes al biomaterial usado, aunque en las reintervenciones para solucionar el daño muscular se encontró cierta dificultad al retirar la malla por las adherencias a la periórbita (Figuras 5 y 6).

La discusión sobre las indicaciones y tiempos de corrección de la fractura orbitaria aún es controversial. El hematoma retrobulbar con compresión del globo ocular o el nervio óptico en combinación con alteración de la visión son señaladas como las indicaciones absolutas e inmediatas ${ }^{30,31}$. Las otras indicaciones incluyen enoftalmo mayor a $2 \mathrm{~mm}$ durante las primeras 6 semanas con un hipoftalmo importante o 

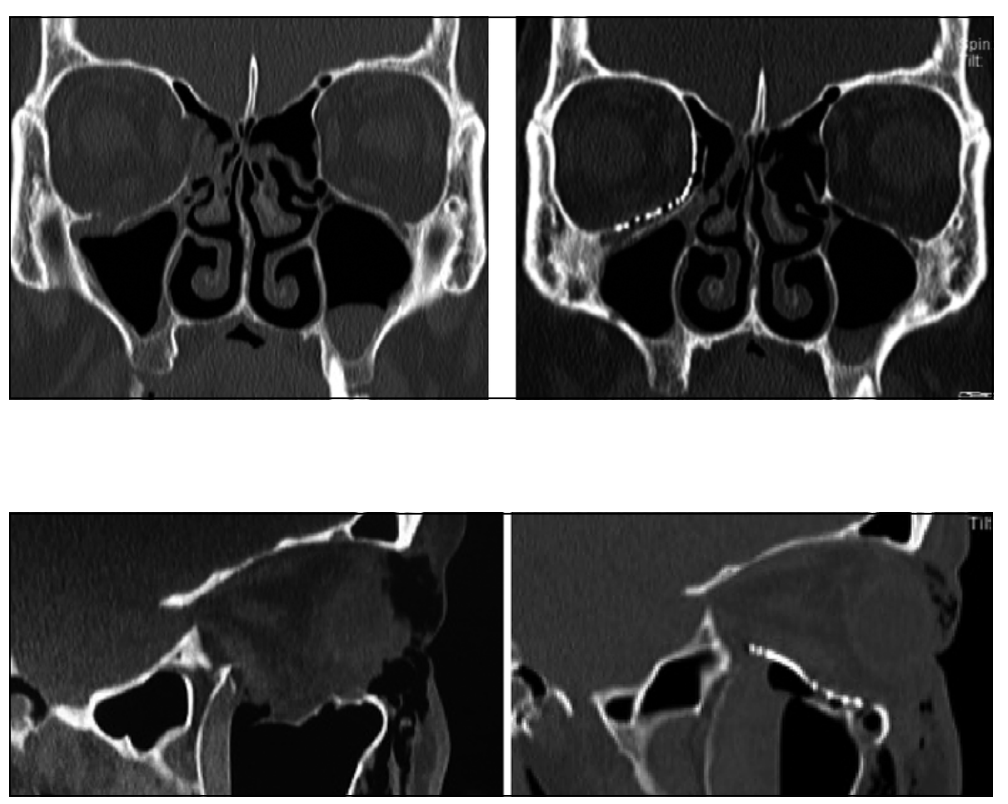

Figura 5. Corte coronal de tomografía computada donde se observa una fractura de piso orbitario asociada a una de pared medial. En el segundo corte se observa la corrección y reconstitución de la anatomía previa al trauma mediante una malla de titanio.

Figura 6. Mismo paciente que imagen 6. Corte sagital que demuestra la caída de tejido herniado hacia el seno maxilar y atrapamiento del músculo recto inferior. En la segunda toma sagital se aprecia la posición de la malla con la presencia de la eminencia retobulbar correctamente adaptada. diplopía, especialmente en el primer campo visual, que no se resuelve antes de las 2 semanas $^{32,33}$. Recientemente la mayoría de los autores han recomendado una cirugía correctiva temprana para mejores resultados y además, para disminuir las incidencias de diplopía y enoftalmos por su menor predisposición a tener tejido blando cicatrizal ${ }^{20,21,34-36}$.

Dejando las indicaciones quirúrgicas iniciales, utilizamos la siguiente evaluación a largo plazo: Nuestro servicio se basa en 3 niveles de diplopía post-trauma, basadas en el reingreso laboral, si el paciente presenta una diplopía como secuela en el grado moderado a severo (leve: diplopía ausente o en mirada mayor a $20^{\circ}$, moderado: diplopía en mirada entre los $10-20^{\circ}$, severo: diplopía en mirada frontal o menor a $10^{\circ}$ ) no puede reingresar en un $100 \%$ a su trabajo y se establece una secuela. Es por esto que ante diplopías severas o en mirada inferior se indica una reoperación. Al igual que en diplopías moderadas o leves con signos de falla en la reconstrucción. Ante alteraciones mínimas o buena reconstrucción esperar 12 semanas hasta 6 meses con tratamiento kinésico, ya que la situación oftalmológica estable se logra entre 9 y 12 meses.

Ante la aparición de un enoftalmo secundario lo primero que debemos hacer es analizar la posición cigomática (arco cigomático, articulación esfenomalar) para eventualmente realizar osteotomías correctoras. Lo cual es un desafío quirúrgico, ya que puede requerir múltiples cirugías. El grupo de Freiburg, en una serie de publicaciones recientes, reportó el uso de planificación preoperatoria asistida por computador, modelos estereolitográficos y navegación intraoperatoria con excelentes resultados ${ }^{8,25-27}$. Nosotros hasta el momento sólo hemos podido contar con modelos estereolitográficos y prótesis "customizadas" para planificar y mejorar nuestros resultados quirúrgicos en pacientes complejos.

Al revisar la literatura nos encontramos con un creciente y continuo uso del acceso transconjuntival en los últimos años, inversamente proporcional al uso del acceso palpebral inferior. El acceso subciliar al parecer se mantiene en el tiempo ${ }^{9}$. El acceso transconjuntival presenta un menor riesgo de ectropión (retracción del párpado) comparado con el subsubciliar, además de presentar una excelente visualización al adicionarse la liberación del ligamento cantal lateral en su porción inferior (contotomía lateral) ${ }^{37,38}$. Pero este uso adicional de la contotomía lateral, según algunos reportes, aumenta la incidencia de entropión o un mal desplazamiento del canto late$\mathrm{ral}^{12,38}$. En algunas ocasiones, como en los pacientes adultos mayores con marcadas arrugas, aún es útil el acceso subtarsal con un menor riesgo de retracción ${ }^{32}$. No encontramos complicaciones producto del acceso en los casos incluidos en el estudio.

En nuestro servicio se recibe una alta prevalencia del trauma medio facial siendo las agresiones y accidentes automovilísticos los factores causales principales. Esto nos entrega una localización de las fracturas orbitarias similar a lo mencionado en la literatura. Nuestros resultados muestran la diplopía como principal complicación inmediata, factible de ser solucionada, cuando es leve, con tratamiento 
kinésico. El último tiempo hemos incrementado el uso del acceso retrocaruncular por entregar una exposición efectiva de la pared medial, hasta el momento con buenos resultados. Por lo que podemos concluir de nuestra actual curva de aprendizaje, que las mallas de titanio son un material eficaz que permite una apropiada adaptación y bajas complicaciones $(6,7 \%)$.

\section{Referencias}

1. Hoffmann J, Cornelius CP, Groten M, Probster L, Pfannenberg C, Schwenzer N. Orbital reconstruction with individually copy-milled ceramic implants. Plast Reconstr Surg. 1998;101:604-12.

2. Rootman J. Basic anatomic considerations. In: Rootman J, editor. Diseases of the orbit. Philadelphia: JB Lippincott 1988;3-18.

3. Hammer B, Prein J. Correction of post- traumatic orbital deformities: operative techniques and review of 26 patients. J Craniomaxillofac Surg. 1995;23:81-90.

4. Miloro M, Ghali GE, Larsen PE, Waite PD. Petersons Principles of Oral and Maxillofacial Surgery. Hamilton, BC Decker Inc. 2004;479-80.

5. Metzger MC, Schon R, Schulze D, Carvalho C, Gutwald $\mathrm{R}$, Schmelzeisen R. Individual preformed titanium meshes for orbital fractures. Oral Surg Oral Med Oral Pathol. 2006;102:442-7.

6. Kozakiewicz M, Elgalal M, Loba P, Komuński P, Arkuszewski P, Broniarczyk-Loba A, et al. Clinical application of 3D pre-bent titanium implants for orbital floor fractures. J Craniomaxillofac Surg. 2009;37:22934.

7. Tang W, Guo L, Long J, Wang H, Lin Y, Liu L, et al. Individual design and rapid prototyping in reconstruction of orbital wall defects. J Oral Maxillofac Surg. 2010;68:562-70.

8. Schön R, Metzger MC, Zizelmann C, Weyer N, Schmelzeisen R. Individually preformed titanium mesh implants for a true-to-original repair of orbital fractures. Int J Oral Maxillofac Surg. 2006;35:990-5.

9. Gosau M, Schöneich M, Draenert FG, Ettl T, Driemel $\mathrm{O}$, Reichert TE. Retrospective analysis of orbital floor fractures complications, outcome, and review of literature. Clin Oral Investig. 2011;15:305-13.

10. Potter JK, Ellis E. Biomaterials for reconstruction of the internal orbit. J Oral Maxillofac Surg. 2004;62:1280-97.

11. Sargent LA, Fulks KD. Reconstruction of internal orbital fractures with Vitallium mesh. Plast Reconstr Surg. 1991;88:31-8.

12. Kontio R, Suuronen R, Salonen O, Paukku P, Konttinen YT, Lindqvist C. Effectiveness of operative treatment of internal orbital wall fracture with polydioxanone implant. Int J Oral Maxillofac Surg. 2001;30:278-85.

13. Rubin PAD, Shore JW, Yaremchuck MJ. Complex orbital fracture repair using rigid fixation of the internal orbital skeleton. Ophthalmology 1992;99:553.

14. Yaremchuck MJ, Del Vecchio DA, Fiala TGS. Microfixation of acute orbital fractures. Ann Plast Surg. 1993;30:385.

15. Ellis E III, Tan Y. Assessment of internal orbital reconstruction for pure blowout fractures: cranial bone grafts versus titanium mesh. J Oral Maxillofac Surg. 2003;61:442-53.

16. Sugar AW, Kuriakose M, Walshaw ND. Titanium mesh in orbital wall reconstruction. Int J Oral Maxillofac Surg. 1992;21:140-4.

17. Mackenzie DJ, Arora B, Hansen J. Orbital floor repair with titanium mesh screen. J Craniomaxillofac Trauma 1999;5:9-16.

18. Kontio R, Lindqvist C. Management of orbital fractures. Oral Maxillofac Surg Clin North Am. 2009; 21:209-20.

19. Lee HB, Nunery WR. Orbital adherence syndrome secondary to titanium implant material. Ophthal Plast Reconstr Surg. 2009;25:33-6.

20. Dutton JJ, Manson PN, Putterman AM, Iliff N. Management of blow-out fractures of the orbital floor. Surv Ophthalmol. 1991;35:279-98.

21. Nam SB, Bae YC, Moon JS, Kang YS. Analysis of the postoperative outcome in 405 cases of orbital fracture using 2 synthetic orbital implants. Ann Plast Surg. 2006;56:263-7.

22. Lee S, Maronian N, Most SP, Whipple ME, McCulloch TM, Stanley RB, et al. Porous high-density polyethylene for orbital reconstruction. Arch Otolaryngol Head Neck Surg. 2005;131:446-50.

23. Gear AJ, Lokeh A, Aldridge JH. Safety of titanium mesh for orbital reconstruction. Ann Plast Surg. 2002;48:1-7.

24. Gellrich NC, Schramm A, Hammer B. Computer-assisted secondary reconstruction of unilateral posttraumatic orbital deformity. Plast Reconstr Surg. 2002;110:141729.

25. Schmelzeisen R, Gellrich NC, Schoen R, Gutwald R, Zizelmann C, Schramm A. Navigation-aided reconstruction of medial orbital wall and floor contour in craniomaxillofacial reconstruction. Injury 2004;35:95562 .

26. Metzger MC, Schon R, Weyer N, Rafii A, Gellrich $\mathrm{NC}$, Schmelzeisen R, et al. Anatomical 3-dimensional pre-bent titanium implant for orbital floor fractures. Ophthalmology 2006;113:1863-8.

27. Metzger MC, Schon R, Zizelmann C, Weyer N, Gutwald R, Schmelzeisen R. Semiautomatic procedure for individual preforming of titanium meshes for orbital fractures. Plast Reconstr Surg. 2007;119:969-76.

28. Kuttenberger JJ, Hardt N. Long-term results following reconstruction of craniofacial defects with titanium micro-mesh systems. J Craniomaxillofac Surg. 2001;29:75-81

29. Park HS, Kim YK, Yoon CH. Various applications of 
titanium mesh screen implant to orbital wall fractures. J Craniofac Surg. 2001;12:555-60.

30. Girotto JA, Gamble WB, Robertson B, Muehlberger T, Mayer M, Zinreich J, et al. Blindness after reduction of facial fractures. Plast Reconstr Surg. 1998;102:1821-34.

31. Gerbino G, Ramieri GA, Nasi A. Diagnosis and treatment of retrobulbar haematomas following blunt orbital trauma: a description of eight cases. Int J Oral Maxillofac Surg. 2005;34:127-31.

32. Cole P, Boyd V, Banerji S, Hollier LH. Comprehensive management of orbital fractures. Plast Reconstr Surg. 2007; 120:57-63.

33. Burnstine MA. Clinical recommendations for repair of orbital facial fractures. Curr Opin Ophthalmol. 2003;14:236-40.
34. Harris GJ Orbital blow-out fractures: surgical timing and technique. Eye 2006;20:1207-12.

35. Ng SG, Madill SA, Inkster CF, Maloof AJ, Leatherbarrow B. Medpor porous polyethylene implants in orbital blowout fracture repair. Eye 2001;15:578-82.

36. Hosal BM, Beatty RL. Diplopia and enophthalmos after surgical repair of orbital fracture. Orbit 2002;21:27-33.

37. Ben Simon GJ, Molina M, Schwarcz RM, McCann JD, Goldberg RA. External (subciliary) vs internal (transconjunctival) involutional entropion repair. Am J Ophthalmol. 2005;139:482-7.

38. De Riu G, Meloni SM, Gobbi R, Soma D, Baj A, Tullio A. Subciliary versus swinging eyelid approach to the orbital floor. J Craniomaxillofac Surg. 2008;36:439-42. 